\title{
Laboreal
}

Volume $5 \mathrm{~N}^{\circ} 1$ | 2009

Ergonomia e desenvolvimento sustentável

\section{Aspectos sociales en el desarrollo sustentable de los sistemas de trabajo : colaboración universidad- sindicatos respecto del trabajo de las mujeres}

Aspectos sociais no desenvolvimento sustentável dos sistemas de trabalho: colaboração universidade-sindicatos relativamente ao trabalho das mulheres Aspects sociaux du développement durable des systèmes de travail: collaboration université-syndicats concernant le travail des femmes Social aspects of the sustainable development of work systems : universitytrade unions collaboration concerning women's work

\section{Ana María Seifert}

\section{OpenEdition}

\section{Journals}

Edición electrónica

URL: http://journals.openedition.org/laboreal/10274

DOI: $10.4000 /$ laboreal. 10274

ISSN: 1646-5237

Editor

Universidade do Porto

Referencia electrónica

Ana María Seifert, « Aspectos sociales en el desarrollo sustentable de los sistemas de trabajo colaboración universidad-sindicatos respecto del trabajo de las mujeres », Laboreal [En línea], Volume $5 N^{0} 1$ | 2009, Publicado el 01 julio 2009, consultado el 10 octubre 2019. URL : http:// journals.openedition.org/laboreal/10274; DOI : 10.4000/laboreal.10274

Este documento fue generado automáticamente el 10 octubre 2019

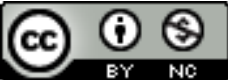

Laboreal está licenciado com uma Licença Creative Commons - Atribuição-NãoComercial 4.0 Internacional. 


\section{Aspectos sociales en el desarrollo sustentable de los sistemas de trabajo : colaboración universidad- sindicatos respecto del trabajo de las mujeres}

Aspectos sociais no desenvolvimento sustentável dos sistemas de trabalho: colaboração universidade-sindicatos relativamente ao trabalho das mulheres Aspects sociaux du développement durable des systèmes de travail : collaboration université-syndicats concernant le travail des femmes Social aspects of the sustainable development of work systems : universitytrade unions collaboration concerning women's work

Ana María Seifert

NOTA DEL EDITOR

Manuscrito recibido en : marzo/2009

Aceptado tras peritage : mayo/2009

\section{Introducción}

Los textos fundadores y los documentos oficiales más conocidos como el informe Brundtland o la Agenda 21 de la conferencia de Rio, definen el desarrollo sustentable como "aquel que satisface las necesidades del presente sin comprometer las necesidades de las futuras generaciones " y hablan de tres dimensiones : la económica, la social y la ecológica. Según Dubois y Mahieu (2002) en cada una de estas dimensiones, 
la sostenibilidad tiene tres constituyentes : la accesibilidad a bienes y servicios, la mantención y el refuerzo de las capacidades para satisfacer las necesidades y la equidad.

2 Transpuesto al medio de trabajo, el desarrollo sustentable en su dimensión social comprende la preocupación por mejorar las condiciones de trabajo y por respetar la vida y la integridad de las personas en el lugar de trabajo. Socialmente, esto debiera traducirse por políticas que protejan de manera equitativa a los y las trabajadoras y que prevengan los problemas de salud y seguridad que puedan afectar sus capacidades. Las empresas son identificadas como actores importantes a causa de las consecuencias de su actividad sobre la naturaleza y porque a través de las condiciones que ofrecen a sus empleados participan a crear o a reducir las desigualdades sociales.

3 Las mujeres trabajadoras enfrentan una situación de desigualdad social en cuanto a la prevención de los problemas de salud relacionados con el trabajo. En los hechos, existe relativamente poco reconocimiento de los riesgos en el trabajo de las mujeres a pesar que las exigencias y los riesgos son diferentes de aquellos presentes en los empleos masculinos. Las mujeres trabajan en sectores económicos, profesiones y puestos diferentes (Messing, Chatigny \& Courville, 1998 ; OIT, 2008), con diferentes horarios y tipos de contratos (Cranford, Vosko \& Zukewich, 2003; Conseil du statut de la femme, 2000 ; Vosko, Zukewich \& Cranford, 2003 ; Vosko, 2000) incluso en la misma ocupación, los hombres y las mujeres no realizan las mismas tareas (Boucher, 1995).

Esta falta de reconocimiento se traduce por una menor accesibilidad a las medidas de prevención y de indemnización de los problemas de salud relacionados con el trabajo. Por ejemplo, en Québec ciertas medidas legales, como ser el nombramiento de un trabajador que se ocupe de la prevención, se aplican únicamente en los tres primeros sectores (sobre ocho) considerados prioritarios. El análisis de la composición de la mano de obra activa muestra que solamente $15 \%$ de las mujeres trabajan en estos sectores (Messing, 2002a). En lo que concierne a la indemnización de los problemas de salud, la falta de reconocimiento de las exigencias y los riesgos ocupacionales se traduce en une menor indemnización de los problemas de salud ocupacional. Por ejemplo, el análisis de las compensaciones por lesiones psicológicas y músculo esqueléticas en Québec muestra que los casos de las mujeres son menos aceptados (Lippel, 1999 ; 2003).

5 La menor accesibilidad a las medidas de prevención y compensación puede contribuir a generar o amplificar los problemas de salud ocupacional, problemas que pueden mermar las capacidades de las trabajadoras temporal o permanentemente y conducirlas a situaciones de vulnerabilidad social, lo que va en contra del desarrollo sustentable.

6 Para contribuir a los esfuerzos por disminuir estas desigualdades es importante realizar investigaciones sobre las exigencias en los trabajos que ocupan las mujeres. Tales investigaciones pueden contribuir a evitar la banalización de los riesgos en los empleos femeninos, a combatir los prejuicios sociales que pueden mermar la capacidad de las trabajadoras de revindicar mejoras en sus condiciones. En este artículo intentaremos mostrar las exigencias suplementarias de condiciones de trabajo que afectan específicamente o mayoritariamente a las mujeres como ser el trabajo a tiempo parcial y el trabajo en equipo, las razones concretas de la resistencia de las trabajadoras frente a transformaciones que aumentan las dificultades en su tarea y revelar las exigencias de la actividad de conciliación trabajofamilia. Comenzaremos por presentar el contexto 
de colaboración en el que realizamos las investigaciones, para luego ilustrar ciertos aspectos sociales del trabajo de las mujeres por medio de datos parciales provenientes de cuatro estudios sobre: cajeras de bancos (Seifert, Messing \& Dumais, 1997), limpiadoras de los hoteles (Seifert \& Messing, 2006), operadoras de teléfono (Prévost \& Messing, 2001) y técnicas en educación especializada (Seifert, 2007).

\section{2. "Lo invisible que hace daño"}

7 Los estudios que presentaré fueron realizados en el marco de una colaboración entre un grupo de investigadoras, del que hago parte, a desarrollar una colaboración con las tres grandes centrales sindicales de Québec. El objetivo del equipo es contribuir a superar estas desigualdades sociales mejorando las condiciones de trabajo y salud de las mujeres y también aportar evidencias que tengan en cuenta sus realidades para que puedan servir como apoyo a la toma de decisiones en orden a transformar las normas y las políticas. Con este fin, investigamos tanto los riesgos ocupacionales como el tratamiento que reciben en caso de indemnización y readaptación después de un accidente o enfermedad profesional.

8 El equipo está compuesto por representantes de condición femenina y de salud laboral de cada una de las centrales sindicales y especialistas en ergonomía, ciencias jurídicas y ciencias sociales. Cada tres años los representantes sindicales consultan a sus bases e instancias, para identificar problemáticas emergentes, a partir de las cuales se definen los proyectos de investigación. Los resultados se presentan tanto a los responsables sindicales como a los trabajadores y trabajadoras en diversos foros.

\section{La metodología empleada en los estudios}

Los estudios fueron efectuados con una metodología que tiene elementos comunes a todos los estudios y particularidades que nos permitieron adaptar el estudio a sus objetivos y al contexto. En este punto, presentaremos les elementos comunes que se refieren al proceso de la investigación, las especificidades de la metodología utilizada serán explicadas en la presentación de los estudios.

El proceso de la investigación comprende varias etapas : 1) formación de un comité de seguimiento ;2) definición y análisis de las necesidades expresadas por las trabajadoras y por el sindicato que pidieron la investigación, así como, por diferentes actores del lugar de trabajo: (supervisores, gerentes, directores, colegas de otras profesiones); 3 ) la observación preliminar complementada con explicaciones "in situ" sobre de la actividad de trabajo; 4) retorno de los resultados preliminares al comité de seguimiento con una propuesta de investigación; 5) observaciones sistemáticas y entrevistas, 6) presentación de los resultados ; 7) informe final, y diseminación de los resultados.

11 La metodología utilizada, característica de las investigaciones en ergonomía, es de corte mayoritariamente cualitativo, pero en varias investigaciones utilizamos también ciertos métodos cuantitativos, por ejemplo, registro y análisis cuantitativo de movimientos en las observaciones o la utilización de cuestionarios con preguntas cerradas. Este tipo de metodología permite analizar en profundidad las dinámicas y las 
relaciones entre los diferentes factores en causa, pero no permite generalizar los resultados a todos los lugares de trabajo y a todas las situaciones.

\section{Los estudios}

\subsection{Cajeras de banco : dar sentido a la resistencia a cambios que deterioran las condiciones de trabajo y las relaciones entre colegas}

12 La resistencia al cambio es un argumento que los directores de empresas utilizan frecuentemente para explicar las quejas de las trabajadoras frente a las transformaciones de sus tareas. El sindicato de cajeras de una institución bancaria solicitó un estudio porque las cajeras (que en Quebec son $92 \%$ mujeres) decían que sus condiciones de trabajo se transformaban y se deterioraban, sin poder discutir con los empleadores. Estos últimos decían que sus quejas eran parte de un fenómeno normal de "resistencia al cambio".

13 La ergonomía y la psicología del trabajo postulan desde hace tiempo (Faverge et al., 1970 ; Grisez, 1982) que este tipo de argumento que recurre a nociones tradicionales de la psicología, "levanta un muro " cerrando la puerta a un análisis que permita cernir mejor el origen de los problemas detectados. Es a partir de esta constatación teóricometodológica que pensamos que el análisis de la actividad podría aportar datos que las ayuden a mostrar cómo la transformación afecta el trabajo y origina dificultades que no son reconocidas.

14 Las cajeras que encontramos al inicio de la investigación explicaron que sus tareas se transformaban del servicio al cliente hacia la venta de productos financieros, mencionaron también que tenían otros problemas importantes como el impacto psicológico de los robos de banco y el estar de pie casi inmóviles durante largas horas.

15 Los ergónomos nos preguntamos si con nuestra metodología podríamos dar respuestas a todas las preocupaciones y colaboramos con una socióloga para documentar las problemáticas identificadas en torno a las ventas, los robos y lo que pasaba después del robo.

16 La investigación comenzó con una serie de cuatro entrevistas colectivas a un grupo de seis cajeras que trabajaban en diferentes situaciones : a tiempo completo o parcial, en sucursales comerciales, de barrio humilde o rico. Se efectuaron ocho observaciones preliminares de 3 a 5 horas en seis sucursales, previa autorización de la dirección. Las observaciones y las entrevistas colectivas permitieron situar el contexto y documentar la organización del trabajo, las principales etapas del trabajo y las estrategias para hacer frente a las dificultades y a los temores ocasionados por los robos. En la etapa sistemática se efectuaron 1) 25 horas de observación del ciclo de trabajo, la frecuencia de ciertas operaciones, 35 horas sobre la postura de pie, su importancia, su repartición a lo largo del día de trabajo, así como los obstáculos a una postura sentada ;2) 18 entrevistas individuales con 11 cajeras, 6 gerentes y un cuadro superior del banco, estas entrevistas permitieron profundizar la percepción de las problemáticas en torno a los robos de banco y al trabajo de venta ; 3) un cuestionario repartido a 547 cajeras en $60 \%$ de las sucursales y respondido por 309 cajeras (54\%). Este cuestionario abordaba los síntomas de problemas musculoesqueléticos, el trabajo de ventas, el número y características de los robos de banco y por el último robo, los sentimientos de la cajera, 
los síntomas de angustia (escala de 14 puntos de Ilfeld) y las ausencias durante tres meses luego del robo.

Los resultados muestran que la mayoría de las cajeras de este banco trabajan en sucursales bancarias pequeñas ( 4 a 10 cajeras) en las que las relaciones interpersonales son próximas. Las observaciones y entrevistas mostraron que estas relaciones son muy importantes tanto para realizar las tareas, como veremos más adelante, como para obtener el apoyo moral de los colegas y supervisores después de los robos de banco. El impacto de los robos es importante, $66,7 \%$ de las cajeras presentan un índice de angustia elevado (escala de Ilfeld) comparado a 30,9\% en la población de mujeres en empleo en 1992-1993 (encuesta salud Québec). En ese contexto, el apoyo es importante para no desequilibrarse frente a los frecuentes robos de banco ya que $72 \%$ de las cajeras que respondieron al cuestionario, fue víctima de al menos un robo y $10 \%$ fue víctima de más de 10 robos. Una cajera decía :

“ Ni mi familia entiende lo que puedo sentir y porqué a veces reacciono días más tarde o lloro. Como la semana pasada, el robo fue tranquilo, sin violencia porque el ladrón vino a mi caja y me pidió el dinero sin mostrar armas, se lo dí y se fue. Pasé el día tranquila, pero cuando llegué a mi casa me puse a llorar y mi esposo me decía "no llores no te hizo nada, estás bien". Por eso es mejor hablar con mis colegas que pueden entenderme y no pensar que exagero ". (traducción de la autora)

18 El servicio al cliente representaba $70 \%$ de su tiempo de trabajo, el resto estaba dedicado a la verificación de operaciones. Los clientes se presentaban al banco y eran dirigidos a una fila única en espera de la primera cajera disponible quien debía recibirlos, realizar las operaciones exigidas e intentar vender productos tales como cartas de crédito, préstamos, seguros, etc.

El trabajo de las cajeras era visto como un trabajo individual, sin embargo, las observaciones muestran que tiene aspectos colectivos. Por ejemplo, en la gestión de la información sobre cambios de procedimiento, cuyo volumen y frecuencia eran altos. No pudiendo memorizar todo, desarrollaron una estrategia colectiva para apoyarse. Se trataba de una especialización, donde cada una retenía la información sobre un tema determinado e informaba o resolvía los problemas sobre este tema. También las ventas eran colectivas porque eran el fruto de la intervención de varias cajeras, ya que los clientes hacían varias visitas antes de decidir una compra y cada vez, podían ser atendidos por una cajera diferente.

La administración impuso objetivos de venta a cada sucursal y a cada cajera. Los resultados de venta de cuatro sucursales muestran que los objetivos no eran alcanzados. Las trabajadoras afirmaban que los objetivos eran irrealistas mientras que la administración sostenía que se resistían al cambio y para estimularlas ofrecieron premios individuales a las ventas.

21 Esta medida fue implantada sin reconocer la naturaleza colectiva del trabajo de venta. Los empleadores subestimaban la complejidad de este tipo de tarea que no se realiza en un solo intento y que es fruto de un esfuerzo colectivo y premiaban solamente la cajera que concretaba el mayor número de ventas. Esta medida, lejos de ayudarlas, debilitaba las relaciones entre colegas, creando malestares y resentimientos. Recordemos que las estrategias de gestión de la información y de preservación de la salud mental de las cajeras se apoyan en las relaciones interpersonales y todo malestar en el grupo, puede desfavorecerlas. 
Esta investigación permitió mostrar la importancia de preservar los colectivos de trabajo y de mostrar que el malestar expresado por las trabajadoras provenía de dificultades reales para realizar la tarea. Varias investigaciones realizadas en el sector bancario en otros países evocan la importancia de preservar los colectivos de trabajo. Por ejemplo, Torres (2006) afirma que "la desvalorización de las sabidurías de experiencia y la demolición de los colectivos, son algunos de los factores que hacen de la banca un espacio con reducidas márgenes de maniobra para la gestión de la actividad y para la preservación de la salud". Palacios, Duarte \& Magalhães Câmara (2002) muestran que en el Brasil, donde la ocupación es mixta, el personal de caja menciona que ciertas agresiones de clientes eran causadas por su exasperación ante la falta de información de los cajeros sobre los productos bancarios. Esta falta de información es debida a la manera como circula la información, que es individual (por medio de comunicados o de instrucciones de los supervisores) y a la falta de reuniones de equipo, lo que imposibilita el desarrollo de estrategias colectivas de memorización de novedades y cambios de procedimientos. Así mismo, los autores señalan que la falta de reuniones de equipo y el poco tiempo de almuerzo va en contra la cohesión de los equipos y favorece las disputas entre colegas. Los autores no analizaron si el hecho de que hayan cajeros y cajeras favorece o dificulta las relaciones entre colegas. Tales estudios van en el mismo sentido que el nuestro y revelan los importantes problemas vividos por cajeros y cajeras. Vemos que ocuparse del trabajo de las mujeres aporta informaciones sobre profesiones que en un país son ocupados por mujeres y que en otros son mixtas y sugiere que las disputas entre colegas no son exclusivas a los lugares de trabajo donde la mano de obra es mayoritariamente femenina.

\subsection{Camareras de los hoteles : revelar las exigencias suplementarias del trabajo a tiempo parcial}

23 En Quebec, $26 \%$ de las mujeres y $12 \%$ de los hombres trabajan a tiempo parcial (Institut de la statistique du Québec, 2007). El trabajo a tiempo parcial es percibido como menos exigente que el de tiempo completo en razón del menor número de horas trabajadas. Sin embargo, en ciertos tipos de empleo, el personal a tiempo parcial tiene condiciones diferentes a las del personal a tiempo completo. Por ejemplo, en los servicios, entran a trabajar en días o en horas de alta afluencia, cumplen sólo ciertas funciones, no reciben toda la información, etc. Las exigencias que les imponen esas condiciones no siempre son reconocidas. Para ilustrar una de estas situaciones presentaremos una investigación que se realizó con las camareras de un hotel de lujo. En la etapa preliminar de la investigación se efectuaron: 1) entrevistas con la gobernanta y el director de recursos humanos ; 2) 32 camareras presentes en un día de trabajo (sobre un total de 40) respondieron a une encuesta que permitió documentar las tareas percibidas como las más difíciles, la localización y la frecuencia de los dolores al final del día de trabajo y las condiciones que las camareras querían cambiar. También se analizaron los informes de accidentes del año en curso para identificar la actividad, las circunstancias en el momento del accidente y el sitio corporal afectado. Los datos sociodemográficos de las trabajadoras fueron documentados a partir de los datos del servicio de recursos humanos.

24 En la etapa sistemática, se analizó : 1) la actividad de trabajo de diez camareras durante 50 horas. Las operaciones consideradas como las más difíciles (hacer la cama, empujar y 
tirar el carro, limpiar el baño) fueron filmadas y sirvieron a un análisis detallado de la frecuencia y de los ángulos posturales de ciertas articulaciones (columna, hombros, muñecas). Durante las observaciones se recabaron informaciones sobre la nacionalidad de la trabajadora y sus estrategias para hacer frente a la sobrecarga de trabajo ; 2) el estado y la carga de cinco carros y documentamos la carga de 20 carros ; 3) la actividad de trabajo del encargado de transportar la ropa a las lencerías durante 16 horas ; 4) la frecuencia de ropa defectuosa, las trabajadoras separaron la ropa defectuosa que encontraban durante un día de trabajo y la investigadora pasó colectando la ropa y la información sobre el número de cuartos limpiados y de camas tendidas por las trabajadoras. Se anotó el número, la forma y el lugar del defecto identificado en cada uno de los artículos de ropa.

Cuando comenzamos, tanto los patrones como el sindicato, afirmaban que las camareras ( $95 \%$ eran mujeres) eran un grupo de edad avanzada, con problemas de salud debidos a su edad, que se quejaba continuamente y que era muy conflictivo. Efectivamente, vimos muchas discusiones sobre las asignaciones de cuartos que muestran que las relaciones de las camareras con los supervisores y entre colegas podían ser muy tensas.

Las camareras de este hotel son inmigrantes y tienen un promedio de 42 años de edad (de 19 a 67) y una antigüedad de diez años (de tres meses a 24 años). El $25 \%$ que tiene más antigüedad (15 años) trabaja a tiempo completo durante todo el año, $75 \%$ trabaja irregularmente, a tiempo completo en estación de alta afluencia turística y al llamado en períodos de menor ocupación. El mecanismo de llamado al trabajo está previsto en el contrato colectivo : se llama por orden de antigüedad y si la trabajadora no está se deja el recado y se debe esperar una hora antes de llamar a la persona siguiente en la lista de antigüedad. Sin embargo, este mecanismo teórico está en realidad sujeto a la discreción de la supervisora que puede o no tener una cierta tolerancia en el tiempo de espera del llamado.

Nos interesamos en la influencia de las asignaciones irregulares de cuartos a limpiar sobre las exigencias físicas del trabajo y sobre las relaciones entre colegas y entre camareras y supervisores.

Las trabajadoras debían limpiar 17 habitaciones por día. Las más antiguas (25\%), tenían asignada una sección de habitaciones contiguas, las otras recibían sus afectaciones el mismo día sin saber de un día para otro si iban a tener las mismas. Las menos antiguas podían verse afectadas a varias secciones alejadas entre ellas o situadas en diferentes pisos, lo que significa que debían trasladarse de una sección a la otra con el carro que lleva la ropa y los productos. Estos carros pesaban entre 170 y 180 kilos al principio del día cuando solo tienen ropa limpia, el peso aumenta en el curso del día cuando la ropa limpia es substituida por ropa sucia, muchas veces húmeda. Además la mayoría de los carros tenía las ruedas defectuosas, lo que aumentaba el esfuerzo físico para empujarlo y provocaba lesiones en las articulaciones.

29 La tarea es muy exigente físicamente y $95 \%$ de las camareras se quejaban de dolores, los principales son dolores de espalda y hombros que afectan a la mitad y al tercio de ellas respectivamente. Las exigencias físicas provocan lesiones que a veces son graves y provocan discapacidades permanentes. Cuando esto ocurre, la trabajadora pierde su empleo porque siendo inmigrante y no dominando el idioma no puede ocupar otros puestos menos exigentes físicamente, en el mismo hotel. La exigencia temporal era alta, porque disponían de 25 minutos por cuarto para hacer las camas (cada habitación 
cuenta con dos camas, tamaño King), limpiar el baño, lavar la vajilla, reponer los productos (café, shampoo), desempolvar los muebles y pasar la aspiradora.

Las camareras que tenían una sección desarrollaron estrategias individuales para regular su carga de trabajo : a) limpiar minuciosamente algunas habitaciones por día y mantener las otras, b) hacer ciertas tareas por adelantado (cambiar la cortina de la ducha, reponer el papel higiénico, etc.) cuando prevén días sobrecargados ; c) omitir aquellas poco visibles, por ejemplo el desempolvado d) reclamar a los supervisores si consideran que la carga de trabajo es excesiva, por ejemplo, si tienen muchas habitaciones en las que el cliente se va, ya que necesitan una limpieza mucho más minuciosa que consume en promedio $60 \%$ más tiempo que una habitación ocupada. Existen también formas colectivas de regulación a través de la ayuda que las amigas ofrecen.

Cuando las trabajadoras no tienen una sección, no pueden regular la carga de trabajo de la misma manera principalmente por tres razones: a) al no tener las mismas habitaciones de un día para otro, no pueden distribuir el trabajo en varios días, ni hacer tareas por anticipado ; b) el temor a no ser llamadas a trabajar hace difícil que reclamen a las supervisoras cuando la carga de trabajo es excesiva (habitaciones de doble ocupación, situadas en pisos diferentes y con clientes que salen) ; c) si omiten tareas, las trabajadoras " titulares" de la sección les reclaman, lo que provoca conflictos.

Los conflictos afectan a las camareras, porque las pueden privar de la ayuda que las colegas podrían darles en los momentos de sobrecarga y de la posibilidad de regular su trabajo discutiendo con los supervisores por temor a ser mal consideradas y a afectar sus posibilidades de ser llamadas a trabajar.

Los resultados fueron presentados en una reunión de 120 representantes de los sindicatos, varias recomendaciones fueron incorporadas a la negociación del contrato colectivo, lográndose una mejora sustancial : la reducción del número de habitaciones a limpiar (de 17 a 14) y del número de habitaciones con clientes que salen (de 12 a 10) así como mecanismos formales de ayuda cuando se prevé una sobrecarga general, por ejemplo, los días en que grandes grupos de clientes se van y otros llegan con horas de diferencia.

Los resultados de esta investigación coinciden con otras investigaciones en el sector hotelero (Lee \& Krause, 2002; Muqa, Mathieu, Sanchez, Roux \& Crestois, 1996), en cuanto a las exigencias temporales y físicas del trabajo de las camareras, sin embargo, nuestra investigación es la única que analizó las exigencias particulares de las camareras que trabajan a tiempo parcial.

El análisis de la actividad mostró que se encontraban en desventaja porque la organización del trabajo-mediante la asignación irregular de habitacioneshacía difícil la utilización de estrategias de anticipación, de distribución de la carga de trabajo en varios días y de omisión de tareas y cuando lo hacían, el costo era alto : el riesgo de ser considerada una "mala trabajadora" o de tener conflictos con el personal a tiempo completo.

Analizar las exigencias especificas del trabajo atípico en el caso de las mujeres es importante puesto que las nuevas formas de organización del trabajo en los sectores fuertemente feminizados como es el sector hotelero, predican la flexibilización de la mano de obra, flexibilización que se traduce en la existencia de "núcleo duro" y minoritario de trabajadores regulares y a tiempo completo y una mayoría del personal 
que trabaja a tempo parcial, al llamado o subcontratado (Bernhardt, Dresser \& Hatton, 2003). Con nuestra investigación mostramos que estas últimas no solo enfrentan una precariedad económica causada por la inseguridad de sus salarios sino también dificultades más importantes que las de las camareras que forman parte del "núcleo duro". Su poder para negociar individualmente cuando la carga de trabajo es excesiva o para no cumplir con la cuota de cuartos a limpiar es menor puesto que, a pesar de las reglas previstas en el contrato colectivo, el llamado a trabajar depende de la "buena voluntad" para que se las llame y se tenga una cierta tolerancia en los tiempos de espera de las respuestas.

\subsection{Telefonistas : hacer visible el trabajo requerido por la conciliación trabajo-familia}

La mano de obra canadiense, que no tiene el mismo horario de trabajo de un día para otro, era de cerca del $30 \%$ en 1994 (Groupe consultatif sur le temps de travail et la répartition du travail, 1994), no encontramos datos más recientes, pero pensamos que esta proporción aumentó a causa de las transformaciones del mercado de trabajo. La variabilidad de los horarios, puede afectar la conciliación trabajo-familia.

Presentaremos un estudio sobre la conciliación trabajo-familia en una central telefónica donde los horarios eran muy irregulares. Generalmente los estudios sobre este tema son realizados por investigadores del área de ciencias sociales. Sin embargo, el análisis de la actividad puede aportar conocimientos complementarios y originales. Las investigadoras, que eran ergónomas, propusieron considerar la conciliación trabajo-familia como una actividad destinada a cumplir paralelamente con las responsabilidades del trabajo y la familia y documentar lo relacionado con las condiciones de trabajo

El estudio comenzó con 12 entrevistas individuales, una entrevista colectiva con seis telefonistas y 16 horas de observaciones de la actividad de trabajo. Esta etapa permitió precisar los elementos a profundizar y caracterizar la organización del trabajo y las tareas. Luego, se procedió a una encuesta que permitió determinar la proporción de telefonistas que tenían niños de 12 años y menos en una gran empresa. Treinta telefonistas de tres divisiones de dicha empresa, cumplían este requisito y aceptaron participar al estudio. Los datos fueron recabados mediante un diario de actividades que las trabajadoras completaban todos los días durante 15 días. Este diario documentaba las actividades que las trabajadoras realizaban para segurar el cuidado de sus hijos (cambios de horario, intercambios de horario con una colega, llamados a las cuidadoras) y la red de personas a las que acudían.

Las telefonistas participantes tenían una antigüedad promedio de 14 años (5 a 19), la mayor parte vivía en pareja. Diez y siete telefonistas tenía uno o varios hijos menores de 6 años, 12 tenían uno o dos niños con problemas de salud que necesitaban vigilancia (alergias o asma) y dos tenían un hijo con problemas graves de salud que necesitaban hospitalización. Los niños tenían una edad promedio de siete años. Los horarios de trabajo eran irregulares y cubrían un período de ocho días. Los horarios eran planificados según la antigüedad, cada semana las telefonistas indicaban sus preferencias en un formulario y su horario le era entregado cuatro días antes de su entrada en función. 
Los resultados muestran que :

a. los horarios eran muy irregulares, incluso para aquellas que tenían más antigüedad. Efectivamente, aún con 18 años de antigüedad, la hora del comienzo podía variar de 10 horas o más de un día para otro ;

b. la red de personas que les cuidaban a los hijos estaba compuesta, en promedio, de cuatro personas (máximo ocho personas). Durante los 15 días, las telefonistas hicieron 212 tentativas (promedio siete, mínimo cinco, máximo 10) para asegurar la disponibilidad de la cuidadora durante sus horarios de trabajo. Estas tentativas se hicieron contactando a la cuidadora, en promedio, un día antes ;

c. las tentativas para cambiar el horario de trabajo eran frecuentes, en los 14 días hicieron 156 tentativas de las cuales $47 \%$ apuntaban a asegurar el cuidado de los niños y el resto a asegurar una vida familiar más coherente.

Las trabajadoras decían sentir mucho stress y deseaban que la variabilidad fuera reducida : “Tengo la impresión que no alcanzo a hacer todo, que si continúo a correr de esta manera, mi corazón va a explotar" "No hay nada peor que cambiar las horas de trabajo, haciendo un up and down, te enfermas ".

Documentando las estrategias utilizadas para asegurar el cuidado de sus hijos y preservar la vida familiar, mostramos que la organización del trabajo aumentaba la complejidad y la importancia de la actividad de conciliación de las telefonistas. Sin embargo, a pesar de su intensidad, esta actividad era invisible para los empleadores. Los horarios irregulares e imprevisibles son una forma de gestión de la mano de obra que cobra importancia. En este contexto, pensamos que es pertinente que los ergónomos consideren ampliar el espectro de sus intervenciones y considerar la actividad de conciliación trabajo-familia como parte de la carga de trabajo.

\subsection{Trabajadoras en educación especializada : hacer visibles las exigencias de coordinación del trabajo colectivo}

Las trabajadoras técnicas en educación especializada (TES) son mujeres en una proporción de $85 \%$. El sindicato pidió un estudio en razón de insatisfacciones y dificultades en el trabajo de equipo.

La investigación comenzó con una entrevista colectiva que reunió cuatro trabajadoras. Los resultados de esta entrevista y el análisis de un documento, producido por el sindicato, sobre las condiciones de trabajo y las dificultades encontradas del documento permitieron definir los temas a tocar durante la investigación y elaborar la guía de observaciones y entrevistas individuales. Luego se documentaron estos temas a través de 10 observaciones de una duración de tres a seis horas, cinco en primaria y cinco en secundaria. Las observaciones fueron efectuadas con TES que trabajan con dos tipos de clientela (problemas de comportamiento, problemas invasivos del desarrollo) y en dos tipos de situaciones (alumnos que cursan sus estudios en clases especiales compuestas por alumnos con el mismo problema y alumnos integrados en clases regulares). Las observaciones permitieron documentar el ciclo de trabajo, las actividades realizadas, los contactos con otros colegas y con los alumnos (frecuencia, tema, lugar, contenido) y caracterizar los locales de trabajo (emplazamiento con respecto a los locales de los otros empleados, adaptación del espacio a las actividades, etc.). Se realizaron 12 entrevistas individuales con las personas observadas y sus colegas de equipo (cuando trabajaban en dupla), las entrevistas permitieron explicitar las problemáticas que se 
constataron y comprender mejor los problemas que las TES vivían. Observaciones más detalladas en dos equipos de trabajo, permitieron documentar la influencia de la frecuencia y el tipo de reuniones entre TES y profesores, sobre el trabajo en equipo y el comportamiento de los alumnos. Entrevistas y observaciones sirvieron a la elaboración de un cuestionario que documentaba la historia laboral (continuidad/ discontinuidad en el puesto, horas de trabajo por año), la percepción de las TES sobre las comunicaciones y la coordinación del trabajo con sus colegas y con los profesores, el acceso a locales necesarios para su trabajo y la percepción sobre la adaptación de tales locales en función de las situaciones de trabajo. El cuestionario fue respondido por 32 TES ( $40 \%$ de las que figuraban en las listas sindicales). Los resultados fueron presentados para su validación y enriquecimiento en la región donde se hizo la investigación y en seis regiones de la provincia de Québec. La validación contó con la participación de 46 personas: TES que trabajaban con diferentes clientelas, profesores y psicoeducadores. Su objetivo era de verificar en qué medida las conclusiones del estudio correspondían a la situación en las otras regiones y discutir con el personal de otras profesiones sobre su percepción de los problemas de coordinación.

Los resultados muestran que la gran mayoría (70\%) se ocupan de alumnos que presentan problemas graves de comportamiento. La antigüedad promedio es de ocho años en la Comisión escolar, sin embargo, 77 \% en primaria y $32 \%$ en secundaria tenían una antigüedad de un año en el puesto ocupado al momento de la investigación. Esta diferencia se debe a que la asignación a un puesto específico se hace una vez por año. Las TES más antiguas tienen la posibilidad de elegir el puesto que más les interese y el resto toma los contratos que quedan. Los contratos más buscados son los de 35 horas por semana, pero $60 \%$ de las TES trabajan con contratos de 30 horas y menos por semana.

En las unidades de educación especializada que acogen alumnos con dificultades graves de comportamiento, las profesoras tienen clases de 12-14 alumnos y son apoyadas por una psicoeducadora que planifica actividades y métodos para enseñar a los alumnos a comportarse en sociedad, y una técnica que se ocupa de aplicarlos en el cotidiano, además de prevenir y controlar las crisis de los alumnos cuando éstas ocurren.

Para trabajar con estos alumnos, es necesario conocerlos, desarrollar una relación de confianza y tener un seguimiento tanto de su comportamiento como de la eficacia de los métodos aplicados para ayudarlos. Las profesoras se ocupan de los alumnos en las clases y las técnicas intervienen si la profesora los expulsa de clases y durante las recreaciones. En este contexto, es fácil entender que las profesoras y técnicas deben conocer la manera de trabajar de la otra y las intervenciones de cada una de ellas deben ser coordinadas. Sin embargo, a causa de la movilidad, al comenzar el año, las profesoras y técnicas no se conocían entre ellas ni conocían a los alumnos. Durante el año, las reuniones eran escasas: $44 \%$ de las técnicas no se reunía nunca con las profesoras, y $39 \%$ lo hacía una vez por mes. La falta de reuniones se debía principalmente a que la mayoría de las TES trabajaba menos de 35 horas semanales, lo que significaba que estaban presentes solamente durante las horas de clases.

La falta de reuniones provocaba una falta de coordinación tanto más importante cuanto tocaba los objetivos de las intervenciones y la adaptación de los métodos de intervención a la evolución de los estudiantes. 59 \% de las TES decían que faltaba coordinación sobre los objetivos y 66 \% sobre la adaptación de los métodos. La falta de coordinación restaba eficacia al trabajo, haciendo difícil la adaptación a la evolución del 
alumno y provocaba serios conflictos entre la profesora y la técnica, conflictos que los empleadores atribuían a problemas de personalidad.

Lavoie (1998) constata que los diferentes tipos de profesionales que intervienen en el campo de la educación especializada (psico-educadores, TES, psicólogos, profesores de gimnasia y de música) tienen su tiempo fragmentado entre varias escuelas por lo que les es difícil tener continuidad y dar servicios satisfactorios tanto a los alumnos como a los profesores. Nuestra investigación permitió ilustrar el impacto de la falta de coordinación y de continuidad en el seno del equipo (profesores, personal técnico y psico-educadores) de las escuelas cuando no se ofrecen espacios de coordinación. Davezies (2005) señala que cuando la organización del trabajo no ofrece posibilidades para una buena coordinación, el personal desarrolla diferentes visiones y criterios de cómo se debe hacer el trabajo, lo que provoca conflictos. Es como si la gestión de la mano de obra se hiciera pensando en el número de horas a cubrir sin tomar en cuenta las exigencias reales de este tipo de trabajo, que necesita tiempo para desarrollar una relación de confianza con el alumno así como continuidad y coordinación en las intervenciones de la TES y de las profesoras. Así, la búsqueda de mayor "eficiencia ", por medio de un ajuste de las horas de trabajo a las necesidades teóricas (Rubery, Ward, Grimshaw \& Beynon, 2005), provoca la pérdida de importantes aspectos del trabajo.

\section{Discusión}

\subsection{El análisis de la actividad y el trabajo de las mujeres}

El análisis del trabajo de las mujeres ha tenido un lugar histórico en el desarrollo de la ergonomía (Teiger et al., 2006). Efectivamente, ya en sus comienzos, varios trabajos permitieron identificar las exigencias del trabajo femenino. Por ejemplo, en trabajadoras de cadenas de montaje (Wisner, Laville \& Richard, 1967), costureras (Teiger \& Plaisantin, 1984), telefonistas (Dessors, Teiger, Laville \& Gadbois, 1978), secretarias a la entrada de datos (Teiger y Bernier, 1992) y en el trabajo doméstico (Doniol-Shaw, 1983). Sin embargo, según Messing (1999), los ergónomos consideraban que hablar del sexo de "los operadores" era una manera reduccionista de tratar la diversidad de las poblaciones. Más recientemente, varias investigaciones ergonómicas se han interesado sea por las especificidades del trabajo en el sector de servicios (Falzon \& Lapeyrière, 1998), fuertemente feminizado, como vimos anteriormente, sea por el trabajo de la mujer de manera explícita, (ver por ejemplo, los importantes trabajos de Messing, de los cuales citaremos el libro "El trabajo de las mujeres : comprender para transformar”, 2002 y los trabajos de Vézina, Tierney \& Messing, 1992; Chatigny, Messing, Laperrière \& Thibault, 2005 ; Bousard, Loriol \& Caroly, 2007).

A partir de nuestra experiencia de colaboración con los sindicatos, sostenemos que es importante interesarse explícitamente en el trabajo de las mujeres. Mostrar sus exigencias y demostrarlas -con hechos muy concretos provenientes del análisis de la actividadpermite que las trabajadoras y sus representantes puedan combatir los prejuicios, no solamente en lo que concierne a los aspectos físicos y mentales del trabajo sino también en lo que concierne a los aspectos sociales, como ser la conciliación trabajo -familia y las relaciones interpersonales en situaciones de trabajo colectivo. 

para cuestionar diferentes aspectos de la organización del trabajo, entre otros las estrategias de gestión que rompen solidaridades (generacionales y en los equipos de trabajo), dificultan la vida familiar y contribuyen a la pérdida del sentido del trabajo, como ser la flexibilización del horario (horarios irregulares e imprevisibles, empleos inseguros, tiempo parcial, etc.) y las transformaciones de la tarea sin tener en cuenta la naturaleza colectiva del trabajo. En una entrevista Caroly (Quéruel, 2008) afirma que analizar el trabajo de las mujeres en profesiones en las que son minoritarias como es el caso de los policías, permite cuestionar la manera como se ejerce la actividad e imaginar otras maneras de hacerlo que pueden también beneficiar a los hombres.

Por estas razones, pensamos que, en América Latina, es importante y pertinente incentivar investigaciones e intervenciones ergonómicas en empleos femeninos, porque de la misma manera que en Canadá, ellas ocupan empleos en sectores diferentes que los hombres, puesto que se encuentran mayoritariamente en los servicios y en el sector informal (OIT, 2008). Su inserción en el mercado de trabajo se hace en condiciones precarias: bajos salarios, empleos inseguros y a tiempo parcial (Parra, 2002). En Venezuela por ejemplo, a principios de los noventa, las mujeres eran $55 \%$ de quienes trabajaban menos de 30 horas semanales (Acevedo, 1997). Aunque esta inserción diferencial en el mercado de trabajo determina un perfil diferente de riesgos en el trabajo, una revisión de los estudios sobre la salud ocupacional en empleos femeninos (Parra, 2002) muestra que se tiende a concebir los problemas de salud de las mujeres como desligados de las condiciones de trabajo. Además, las características de las mujeres tampoco se toman en cuenta en América latina a la hora de diseñar puestos de trabajo o herramientas. Por ejemplo, Gutiérrez y Apud (1996) mostraron que los puestos de trabajo en una procesadora de pescado no consideraban las medidas antropométricas de las trabajadoras chilenas.

\subsection{La importancia de la colaboración con los sindicatos}

En ergonomía de la actividad, se privilegia la creación de un proceso de diálogo y concertación entre los diferentes actores de la empresa por medio de equipos-proyecto mixtos con la presencia de trabajadores, profesionales y cuadros de gestión según la estructura y las condiciones existentes en cada empresa. Se postula que este proceso permite -en la confrontación de diferentes puntos de vista sobre el trabajola evolución de las representaciones del trabajo de los diversos actores y por ende, abre las puertas del cambio (Guérin, Laville, Daniellou, Duraffourg \& Kerguelen, 1997).

Sin embargo, las posibilidades de transformación no dependen solamente del intercambio y concertación que el ergónomo pueda crear en la empresa, ni de la calidad de su trabajo, que puede revelar los costos de los problemas de funcionamiento del proceso productivo y los problemas de salud del personal. Las posibilidades de cambio dependen también de la presión social ejercida por los sindicatos u otros grupos organizados de defensa de los y las trabajadoras, así como de la existencia de normas puesto que en el ámbito del trabajo existen tensiones innegables entre los intereses económicos y los valores sociales como el derecho a un trabajo saludable y al mejoramiento de las situaciones de trabajo.

Estando presentes en los lugares de trabajo, los ergónomos nos encontramos involucrados de lleno en esas tensiones. Según Davezies (1998), frente a los problemas 
actuales en el mundo del trabajo y a la necesidad de mejorar las situaciones de trabajo, el ergónomo puede tener dos roles: lograr que los niveles jerárquicos se interroguen sobre la organización del trabajo o ayudar al personal a ocupar individual y colectivamente un espacio de debate y democracia para lograr el mejoramiento de las situaciones problemáticas.

Sin embargo, la presión social es débil en el caso de los problemas de salud ocupacional de las mujeres que son relativamente poco conocidos o poco reconocidos tanto por las instituciones que dictan las leyes y reglamentos de salud ocupacional como por las centrales sindicales y en última instancia por las trabajadoras mismas (Messing, 2000, 2002a). Por ello, a colaboración con los sindicatos es una de las formas de apoyo que permite abrir debates sobre las problemáticas relacionadas con el trabajo y apoyar el cambio. Los representantes sindicales pueden convertirse en relevos y motores de cambio necesarios a la transformación del trabajo (Teiger, 1993). La colaboración en las investigaciones, transforma sus representaciones del trabajo de las mujeres lo que permite que puedan representarlas mejor en las diferentes instancias en las que están presentes, sean éstas la defensa de sus demandas de indemnización, negociaciones de contratos colectivos o consultas gubernamentales sobre elaboración o evaluación de normas y políticas. Así, los ergónomos pueden jugar un rol importante apoyando-por medio de la generación de conocimientos sobre el trabajo de las mujeresla emergencia de un debate que permita crear una presión social y en última instancia, superar esta situación de desigualdad social.

La colaboración con los representantes sindicales permite también acceder a situaciones y fenómenos a los que los ergónomos no siempre tienen acceso y a acumular investigaciones y estudios de caso en torno a una problemática permitiendo una mirada global y una reflexión social. Aparte de nuestra experiencia existen otros ejemplos, entre ellos citaremos la investigación-acción sobre las consecuencias de la intensificación del trabajo en Francia (Bourdon et al., 2006), en la que un equipo universitario sindical documentó un gran número de situaciones, que puestas juntas, permiten una mirada global sobre esta problemática y el desarrollo de acciones colectivas sobre las consecuencias de la intensificación del trabajo. Estos ergónomos llaman, de la misma manera que nosotros, a reconstruir los lazos con los representantes sindicales.

\section{Conclusión}

Para lograr un desarrollo sustentable de los sistemas de trabajo es necesario investigar los problemas ocupacionales de la mujer. Estas investigaciones contribuyen a romper la invisibilidad de sus problemas y a empoderar a sus representantes para que puedan mejorar las situaciones de trabajo y defenderlas mejor. Investigar el trabajo de las mujeres, lejos de crear divisiones entre trabajadoras y trabajadores contribuye a mostrar los problemas en oficios y profesiones que son ocupados por mujeres en ciertos países y por hombres en otros o que son mixtos, como es el caso del personal bancario y de la educación especializada o a abrir posibilidades de cuestionar las prácticas y transformarlas.

61 Los conocimientos que se generan permiten espacios de reflexión tanto académicos como sociales. En el ámbito de la ergonomía, las investigaciones sobre puestos de trabajo ocupados mayoritariamente por mujeres permitieron ampliar la mirada del 
ergónomo, que partiendo del trabajo, aplicó el marco de análisis de la ergonomía sobre la actividad de conciliación entre el trabajo y la familia. El conjunto de investigaciones y la discusión con las sindicalistas permitió una mirada global sobre las formas de organización del trabajo que destruyen los colectivos de trabajo y provocan conflictos.

\section{BIBLIOGRAFÍA}

Acevedo, D. (1997). Género y flexibilización del trabajo. Impacto en la salud. Cuadernos Mujer Salud, 2, 28-36.

Bernhardt, A., Dresser, L. \& Hatton, E. (2003). The coffee pot wars : Unions and firm restructuring in the hotel industry. In E. Appelbaum, A. Bernhardt \& R. Murnane (Eds), Low Wage America : How Employers Are Reshaping Opportunity in the Workplace (pp 33-76). New York : Russell Sage Foundation.

Boucher, M. (1995). Analyse de l'activité des jardiniers et des jardinières cols bleus. Memoria de maestría en biología. Départament de sciences biologiques. Université du Québec à Montréal.

Bourdon, F., Daniellou, F., Davezies, P., Dugué, B., Gaudart, C., Mezzarobba, D. \& Théry L. (2006). Quand les ergonomes et les syndicalistes renouent les liens. Quelques enseignements d'une recherche-action sur l'intensification du travail. Actes du congrès de la SELF 2006.

Bousard, V., Loriol, M. \& Caroly, S. (2007). Une féminisation sur fond de segmentation genrée : le cas de policières en commissariat. Sociologies pratiques, 14, (1), 75-88.

Chatigny, C., Messing, K., Laperrière, E. \& M.-C Thibault. (2005). Battle fatigue : Identifying stressors that affect counsellors in women's shelters. Canadian Woman Studies, 24, (1), 139-144.

Conseil du statut de la femme (2000). Emploi atypique cherche normes équitables. Québec : Conseil du Statut de la femme.

Cranford, C. J., Vosko, L.F. \& Zukewich, N. (2003). The gender of precarious employment in Canada. Relations industrielles, 58,(3), 454-482.

Davezies, P. (1998). Recherche en ergonomie et santé. Deuxièmes journées Recherche et ergonomie. Toulouse. http :www.ergonomie.self.org/rechergo98/html/Davezies.html

Davezies, P. (2005). La santé au travail, une construction collective. Dossier : Coopérations dans le travail construisent la santé. Travail et Santé, 52, 24-28.

Dessors, D., Teiger, C., Laville, A. \& Gadbois, C. (1978). Conditions de travail des opératrices des renseignements téléphoniques et conséquences sur leur vie personnelle et sociale. Arch. mal. prof, vol. 40, no. 3-4, 469-500.

Doniol-Shaw, G.(1983). L'ergonomie du travail ménager. Groupe d'étude de la division sociale et sexuelle du travail. Paris : Conseil national de la recherche scientifique.

Falzon, P. \& Lapeyrière, S. (1998). L'usager et l'opérateur : Ergonomie et relations de service. Le travail humain, 61, 1, 69-90. 
Faverge, J.M., Hoyoux, M., Olivier, M., Querton, A., Laporta, J. \& Salengros, M. (1970). L'organisation vivante : comportements d'ajustement et d'évolution au sein des organisations. Bruxelles : Éditions de l'Institut de Sociologie.

Groupe consultatif sur le temps de travail et la répartition du travail (1994). Rapport du Groupe consultatif sur le temps de travail et la répartition du travail. Ottawa : Développement des ressources humaines.

Grizes, J. (1982). Ditez-moicequ'ilestimportantdechercher. Númeroespecial de homenaje a JeanMarie Faverge. Le travail humain, 45, (1), 73-77.

Guérin, F., Laville, A., Daniellou, F., Duraffourg, J. \& Kerguelen, A. (1997). Comprendre le travail pour le transformer. Lyon, France : ANACT.

Gutierrez, M. \& Apud, E. (1996). Técnica de maniquíes aplicada a la evaluación de puestos de trabajo operadas por mujeres. Cuadernos Médico-Sociales, 37, (4), 37-47.

Institut de la Statistique du Québec (2007). Caractéristiques du marché du travail selon le sexe, par région métropolitaine de recensement et ensemble du Québec, 2007. Consultado el 25 de septiembre 2008 de : http://www.stat.gouv.qc.ca/donstat/societe/march_travl_remnr/ parnt_etudn_march_travl/pop_active/stat_reg/rmr_sexe_2007.htm Lavoie, G. (1998). Prévalence, intégration et soutien apporté aux élèves présentant des difficultés d'ordre comportemental et à leur enseignant. Consultado el 15 de octubre 2008 de : http://www.

adaptationscolaire.org/themes/dico/documents/prevalence.pdf. Lee, P.T. \& Krause, N. (2002). The Impact of a Worker Health Study on

Working Conditions. Journal of Public Health Policy, 23, (3), 268-285. Lippel, K. (2003). Compensation for musculoskeletal disorders in Quebec : systemic discrimination against women workers? International Journal of Health Services, 33, (2), 253-281.

Lippel, K. (1999). Workers compensation and stress. Gender and access to compensation. International Journal of Law and Psychiatry, 22, (1), 78-89.

Messing, K., Chatigny, C. \& Courville, J. (1998). 'Light' and 'heavy' work in the housekeeping service of a hospital. Applied Ergonomics, 29, (6), 451-459.

Messing, K. (2000). La Santé des Travailleuses : La Science est-elle Aveugle ? Toulouse : Octarès. Messing, K. (2002a). La place des femmes dans les priorités de recherche en santé au travail au Québec. Relations industrielles, 57, (4), 660-686.

Messing, K. (Dir.) (2002b). El trabajo de las mujeres : comprender para transformar. ISTAS-Bureau Technique Syndical Européen pour la Santé et la sécurité.

Messing, K. (1999). La pertinence de tenir compte du sexe des "opérateurs" dans les études ergonomiques : Bilan de recherches. PISTES, 1, (1). http://www.pistes.uqam.ca/v1n1/articles/ v1n1a5.htm Muqa, F., Mathieu, F., Sanchez, M.I., Roux, F. \& Crestois, M. (1996). La femme de chambre. Cahiers de Médecine interprofessionnelle, 3, 305-316.

Organización Internacional del Trabajo (2008). Tendencias mundiales del empleo de las mujeres. Ginebra : OIT. http://www.oei.es/pdfs/wcms_091227.pdf (27 de septiembre 2008)

Palacios, M., Duarte, F. \& de Magalhães Camara, V. (2002). Trabalho et sufrimiento de caixas de agencias bancárias na cidade do Rio de Janeiro. Cadernos de Saúde Pública, Rio de Janeiro, 18, (3), 843-851. Parra, M. (2002). La salud de las mujeres trabajadoras en América Latina. In K. Messing (Dir.), El trabajo de las mujeres : comprender para transformar (pp. 69-128). ISTAS-Bureau Technique Syndical Européen pour la Santé et la sécurité. 
Prévost, J. \& Messing, K. (2001). Stratégies de conciliation d'un horaire de travail variable avec des responsabilités familiales. Le travail humain, 64, 119-143.

Quéruel, N. (2008). Quand le travail malmène les femmes. Santé et travail, 63, 6-8.

Rubery, J., Ward, K., Grimshaw, D. \& Beynon, H. (2005). Working Time, Industrial Relations and the Employment Relationship. Time and Society, 15, 1, 89-111.

Seifert, A. M., Messing, K. \& Dumais, L. (1997). Star wars and strategic defense initiatives : Work activity and health symptoms of unionized bank tellers during work reorganization.

International Journal of Health Services, 27, (3), 455-477.

Seifert, A.M. \& Messing, K. (2006). Cleaning up after globalization : An Ergonomic Analysis of Work Activity of Hotel Cleaners. Antipode : A Radical Journal of Geography, 38, (3), 557-578.

Seifert, A.M. (2007). Is Work in Education Child's Play ? Understanding Risks to Educators Arising from Work Organisation and Design of Work Spaces. New Solutions : A Journal of Environmental and Occupational Health Policy, 17, 97-110.

Teiger, C. Barbaroux, L., David, M., Duraffourg, J., Galisson, M.T., Laville, A., \& Thareaut, T. (2006). Quand les ergonomes sont sortis du laboratoire.... à propos du travail des femmes dans l'industrie électronique (1963 1973). Rétro-réflexion collective sur l'origine d'une dynamique de coopération entre action syndicale et rechercheformation-action. PISTES, 8, (2) http://www.pistes.uqam.ca/ v8n2/ articles/v8n2a4.htm

Teiger, C. (1993) Analyse ergonomique du travail et formation. Colloque "Recherches pour l'ergonomie ". Toulouse.

Teiger, C. \& Plaisantin, M.-C. (1984). Les contraintes du travail dans les travaux répétitifs de masse et leurs conséquences sur les travailleuses. In J. A. Bouchard (Dir), Les effets des conditions de travail sur la santé des travailleuses (pp. 33-68). Confédération des syndicats nationaux, Montréal.

Teiger, C. \& Bernier, C. (1992). Ergonomic analysis of work activity of data entry clerks in the computerized service sector can reveal unrecognized skills. Women and Health, 18, 3, 67-78.

Torres, I. (2006). Cambios organizacionales, salud y bienestar en la banca portuguesa. Edad y sentidos del trabajo en una nueva relación salarial (resúmen). Laboreal, 2, (2), 62-65. http:// laboreal.up.pt/revista/artigo.php?id=37t45nSU547112278541758951.

Vézina, N., D. Tierney, \& K. Messing (1992). When is light work heavy ? Components of the physical workload of sewing machine operators which may lead to health problems. Applied Ergonomics, 23, 268.

Vosko, L., N. Zukewich \& C. Cranford (2003). Le travail précaire : une nouvelle typologie de l'emploi. Perspective, octobre, 17 (Statistique Canada - no 75-001-XIF au catalogue).

Vosko, L. (2000). Temporary Work : The Gendered Rise of a Precarious Employment Relationship. Toronto : University of Toronto Press.

Wisner A., Laville A. \& Richard E. (1967). Conditions de travail des femmes O. S. dans la construction électronique. Laboratoire de Physiologie du Travail et Ergonomie du CNAM. Rapport No. 2. 


\section{RESÚMENES}

El desarrollo sustentable de los sistemas de tra-bajo no se puede realizar sin el bienestar de los trabajadores y trabajadoras. Este artículo plantea la importancia de preocu-parse específicamente del trabajo de las mujeres. Por medio de datos parciales de cuatro estudios realizados en el marco de una experiencia de colaboración con tres grandes centrales sindicales de Québec, ilustramos ciertos aspectos sociales del trabajo que afectan específicamente o mayoritariamente a las mujeres. La metodología se basa en entrevistas y observacio-nes de la actividad del trabajo y es de tipo participativo. A partir de estos estudios, mostramos que el análisis del trabajo de las mujeres y de la conciliación entre el trabajo y la familia cues-tionan las formas de organización del trabajo que debilitan los colectivos de trabajo y afectan las estrategias de regulación de la tarea y de preservación de la salud mental. Discutimos tam-bién la importancia de la colaboración con los sindicatos que aportan una reflexión sobre problemáticas emergentes, difun-den los resultados y contribuyen a generar una presión social necesaria al mejoramiento de las condiciones de trabajo.

O desenvolvimento sustentável dos sistemas de trabalho não pode realizar-se sem o bem-estar dos trabalhadores e trabalhadoras. Este artigo afirma a importância da preocupação específica com o trabalho das mulheres. Através de dados parciais de quatro estudos realizados no âmbito de uma experiência de colaboração com três grandes centrais sindicais do Quebeque, ilustram-se alguns aspectos sociais do trabalho que afectam especificamente ou maioritariamente as mulheres. A metodologia baseia-se em entrevistas e observações da actividade de trabalho e é de tipo participativo. A partir destes estudos, mostramos que a análise do trabalho das mulheres e a conciliação entre o trabalho e a família questionam a forma de organização do trabalho que debilitam os colectivos de trabalho e afectam as estratégias de regulação da tarefa e de preservação da saúde mental. Discutimos também a importância da colaboração com os sindicatos, que aportam uma reflexão sobre problemáticas emergentes, difundem os resultados e contribuem para gerar uma pressão social necessária para a melhoria das condições de trabalho.

Le développement durable des systèmes de travail n'est pas possible sans le bien-être des travailleurs et travailleuses. L'article traite de l'importance de se préoccuper spécifiquement du travail des femmes. Des données partielles de quatre études réalisées dans le cadre d'une expérience de collaboration avec les trois grandes centrales syndicales du Québec sont utilisées pour illustrer certains aspects sociaux $d u$ travail qui affectent spécifiquement ou majoritairement les femmes. La méthodologie est de type participatif et recourt à des entrevues et des observations de l'activité de travail. Les résultats montrent que l'analyse du travail des femmes et de l'activité de conciliation travail-famille contribuent au questionnement des formes d'organisation du travail qui affaiblissent les collectifs et affectent les stratégies de régulation de la tâche et de préservation de la santé mentale. Nous discutons aussi de l'importance de la collaboration avec les syndicats parce que ceux-ci apportent leurs réflexions sur des problématiques émergeantes, s'associent à la diffusion des résultats et contribuent à générer une pression sociale qui est nécessaire pour améliorer les conditions de travail.

Sustainable development of work systems is not attainable without workers' well-being. This article addresses the importance of taking into account women's work. Partial results from four studies carried out within a collaborative project with the three major Quebec labour federations serve to illustrate several social factors that affect women, either exclusively or in the majority. The methodology used is participative, with interviews and workplace observations. The results show how the analysis of work performed by women and the methods they use to facilitate workfamily balance contribute to a calling into question of forms of work organization that weaken work collectives and affect strategies to control work and to preserve mental health. We also 
discuss the importance of working with unions, as they contribute to the identification of emerging issues, to the dissemination of research results and to generating social pressure to improve working conditions.

ÍNDICE

Palabras claves: trabajo de las mujeres, organización del trabajo, conciliación trabajo-familia, trabajo colectivo, conflictos en el trabajo

Keywords: women's work, work organisation, work-family balance, collective work, conflicts at work

Palavras-chave: trabalho das mulheres, organização do trabalho, conciliação trabalho-família, trabalho colectivo, conflitos no trabalho

Mots-clés: travail des femmes, organisation du travail, conciliation travail famille, travail collectif, conflits au travail

\section{AUTOR}

\section{ANA MARÍA SEIFERT}

CINBIOSE Universidad de Québec, Montreal Case postale 8888, Canada H3C 3P8

seifert.ana-maria@uqam.ca 